Bull. Egypt. Soc. Physiol. Sci. Vol. (42) Issue (1), 52-62

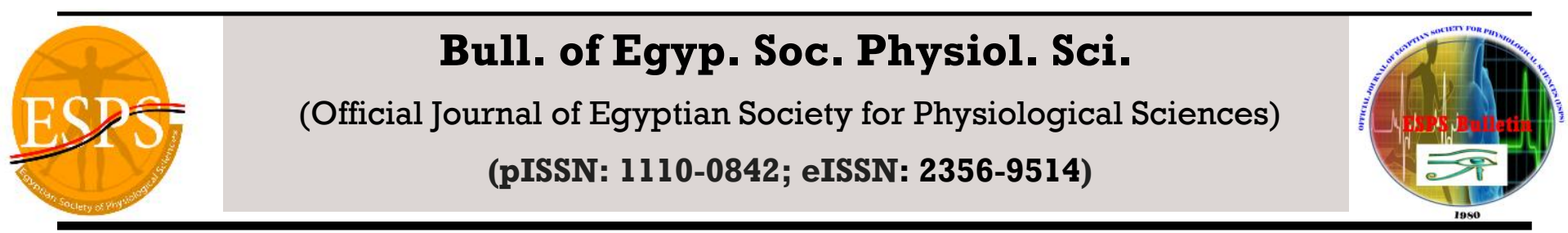

\title{
Elevated body fat percentage as a predictor of irregular ovulation and menstruation
}

\author{
Nesrine M. El Azhary ${ }^{1}$, Nermine El Beltagy ${ }^{2}$, Ahmed M. El Habashy ${ }^{3}$, Nadira M.El Shaer ${ }^{4}$, \\ Sara H. Hassan ${ }^{5}$ \\ ${ }^{1}$ Associate professor of Medical Physiology, Faculty of Medicine, Alexandria University \\ ${ }^{2}$ Professor of Obstetrics and Gynecology, Faculty of Medicine, Alexandria University. \\ ${ }^{3}$ Associate professor of Obstetrics and Gynecology, Faculty of Medicine, Alexandria University \\ ${ }^{4}$ Assistant specialist of Obstetrics and Gynecology, Ministry of Health Hospitals, Alexandria, Egypt \\ ${ }^{5}$ Research assistant, Alexandria University
}

Submit Date:14 June, 2021

Revise Date: 7 July 2021

Accept Date: 14 July 2021

Keywords

Kisspeptin

- Fat percentage

- Irregular ovulation.

\begin{abstract}
Introduction: Excess body fat has been implicated in mediating irregular ovulation and menstruation. The aim of the current work was to study the potential impact of body fat percentage on the serum level of kisspeptin hormone and on the size of the ova, at day 11 of the menstrual cycle in females. Patients and Methods: The study was performed on 80 females, distributed into 3 groups. Group I (control): 20 females with regular menstrual cycles; Group II: 30 females with BMI < 30, with irregular menstrual cycles and Group III: 30 females with BMI $\geq 30$ with irregular menstrual cycles. Females were subjected to detailed history taking, examination and investigations. A food frequency questionnaire was filled. Results: In patients having irregular menstruation (Group II and III), body fat percentage was elevated compared to the control group. They also had more abdominal adiposity. Their mean serum Kisspeptin level as well as the mean size of their ovarian follicles on day 11 were significantly lower compared to group I . Group II and III patients also had a significantly high intake of carbohydrates and a significantly lower intake of vegetables and fruits, compared to group I females. Conclusion: High body fat percentage more than $32 \%$ might contribute to irregular ovulation via decreasing kisspeptin secretion and consequently reducing the size of ovarian follicle at day 11 of the menstrual cycle.
\end{abstract}

Corresponding author: Dr. Nesrine M.El Azhary, Medical physiology department, Mowassah Campus, Faculty of Medicine, Alexandria, Egypt. E-mail: mouniryousef2005@yahoo.fr 


\section{INTRODUCTION}

Obesity means excessive fat accumulation. This extra fat has a negative impact that extends to the reproductive potential. There is an alteration of the HPO axis and higher circulating levels of insulin, which increase ovarian androgen production [1]. Obesity is also associated with anovulation, menstrual disorders and infertility. A small weight loss in anovulatory obese infertile women was found to improve ovulation and pregnancy rate [2]. It has been estimated that $75 \%$ of infertile overweight or obese women have polycystic ovarian syndrome [3]. Obesity has been defined by the WHO as having a body mass index $\geq 30 \mathrm{~kg} / \mathrm{m}^{2}$ [4]. Recently, more methods have been developed to quantify obesity as the relative fat mass that predicts the body fat percentage, a suggested more accurate indicator of obesity $[5,6]$.

Kisspeptin (Kp, previously known as metastin) is a protein encoded by the KISS- 1 gene in humans. Kisspeptins were originally characterized as potent anti-metastatic agents in breast cancer and malignant melanoma cells. Then, they were found to orchestrate several functions as regulating the onset of puberty, mainly through the full activation of HPO axis and generation of cyclical LH surges. GnRH stimulates the pituitary gland to release FSH and $\mathrm{LH}$, which stimulates the follicular growth and begin to produce estradiol, which in turn, inhibits the HPG axis [7]. In late follicular phase, when the main follicle reaches the proper mature size, the level of estradiol increases and stimulates the kisspeptin secreting neurons which have an estradiol receptor to release kisspeptin. i.e., Kp neurons stimulate the $\mathrm{GnRH}$ neurons that induces the LH release, ovulation and corpus luteum development. Corpus luteum produces progesterone, estradiol and inhibin A that inhibit the HPG axis. Kisspeptin directly promotes the secretion of LH and growth hormone in the pituitary gland. In addition, $\mathrm{Kp}$ receptors are expressed in the ovaries, where kisspeptin is involved in the regulation of ovarian progesterone synthesis [8]. Furthermore, kisspeptin also participates in follicle development and ovulation. Clinically wise, the injection of kisspeptin-54 induces egg maturation in women undergoing invitro fertilization therapy [9].

Prediction of ovulation is gaining lots of interest as it is needed for harvesting of ova in females undergoing assisted reproductive techniques [10]. LH surge is a practical marker for ovulation and the urinary LH home test has been widely used in ovulation prediction. However, this elevation is transient and does not reflect the degree of development of the dominant follicle [11].

The aim of the current work was to study the potential impact of body fat percentage on the serum level of kisspeptin hormone and on the size of the ova, at day 11 of the menstrual cycle in females.

\section{Patients and Methods}

After approval of the Ethics Committee of the faculty of Medicine, Alexandria University, this study was conducted in the Medical Physiology department and the department of Obstetrics and Gynecology, EL Shatby University Hospital, over the period of one year. The study included 80 females. Every patient signed a written informed consent. 
Inclusion criteria: Married females aged 20-35 years, who failed to get pregnant after 1year of marriage due to irregular cycles (less than 21 days or more than 35 days) [12], except for the healthy control group who had regular cycles, not using any type of contraception for at least 6 months before participation in the current study and attending the Shatby Hospital for follow up of ovulation.

Exclusion criteria: Married females aged below 20 years or above 35 years, who failed to get pregnant after 1year of marriage due to causes other than irregular cycles (as endometriosis, tubal problems....), taking drugs for induction for ovulation, or using any type of contraception during the last 6 months.

Females participating in the study were allocated into 3 groups:

Group I (control group): 20 females with regular menstrual cycles.

Group II: 30 females with BMI $<30$, with irregular menstrual cycles.

Group II: 30 females with $\mathrm{BMI} \geq 30$ with irregular menstrual cycles.

They were subjected to:

1. Detailed history taking. (age, occupation, duration of menstrual troubles and obstetric history).

2. Clinical examination: General examination and gynecologic examination (abdominal and pelvic) to exclude any pelvic pathology.

3. Investigations:

a) Measurement of weight, height and body mass index (BMI) . Weight in kilograms was assessed using a digital scale. Patients were wearing light clothing, without shoes, after voiding of urine, following minimum of a 4-hour fast. Height was measured to the nearest centimeter using a stadiometer.

b) Body composition analysis by bioelectric impedance using

In Body-220 (Biospace, South Korea), to determine the percentage of body fat [13]

c) To detect abdominal obesity, waist/hip ratio and waist /height ratio

were assessed [14]

d) Serum level of kisspeptin by ELISA technique, according to the manufacturer's instructions [15]

e) Serial Transvaginal Ultrasonography done in the day 11 of menstrual cycle to detect the size of the follicular development, using Mindray ultrasound IBE-2500D apparatus.

f) Filling a food frequency questionnaire about dietary habits. It included the carbohydrate, fat, protein intake as well as vegetables, fresh fruits, dairy intake, carbonated water and juice. Servings were calculated according to exchange lists published by the American dietetic association [16].

\section{Statistical analysis [17]}

Data were fed to the computer and analyzed using IBM SPSS software package version 20.0. (Armonk, NY: IBM Corp). Quantitative data were described using mean and standard deviation. For normally distributed quantitative variables, $\mathrm{F}$ test (ANOVA) was used to compare between more than 2 groups, and Post Hoc test (Tukey) for pairwise comparisons. Spearman coefficient was used to correlate between 2 quantitative variables. For the food frequency questionnaire, Kruskal Wallis test was used to compare between more than two studied 
groups and Post Hoc (Dunn's multiple comparisons test) for pairwise comparisons. Chisquare test was also used to compare between different groups. A value of $\mathrm{P}<0.05$ was considered statistically significant.

\section{Results}

The BMI of group III patients was significantly higher than that of both group I and group II. $(\mathrm{P}=$ 0.001). No statistically significant difference was found between the BMI of group II and group I (control) $(\mathrm{p}=0.817)$ (table 1).

There was no statistically significant difference in age between group I and group II patients as well as between group I and III females. However, group III females had a significantly higher age than group II females $(\mathrm{p}=0.041)($ table 1$)$.

The mean body fat percentage was $28.59 \pm$ $2.03,39.41 \pm 4.29$ and $48.40 \pm 4.53$ in group I, II and III females respectively. i.e. It was significantly higher in both group II and III patients compared to group I control $\left(\mathrm{p}_{1}<0.001\right)$. In addition, group III females had a significantly higher body fat percentage than group II females $(\mathrm{p}<0.001)$ (Figure 1 B). Females with regular menstrual cycles had a body fat percentage $<32 \%$, while in those with irregular menstruation, it was $>32 \%$.

In group II and III patients, the waist/hip ratio was significantly higher than group I females $(p<0.001)$. The waist to height ratio was also significantly higher in group II and III than group I females $(\mathrm{p}<0.001)$ (table 1$)$.

The mean Kisspeptin level on day 11 was $349.3 \pm 73.69,227.0 \pm 71.86$ and $227.0 \pm$ $71.86 \mathrm{pg} / \mathrm{mL}$ in group I, II and III respectively. It was significantly lower in group II and III compared to group I (control) $(\mathrm{p}=0.001)$. No statistically significant difference were found between group II and III $(\mathrm{p}=0.288)$ (Figure $2 \mathrm{~A})$.

The mean size of the ovarian follicle on day 11 of the menstrual cycle of the participating females was $11.65 \pm 0.49,7.07 \pm 0.78$ and $7.0 \pm$ $0.79 \mathrm{~mm}$ in group I, II and III respectively. It was significantly lower in group II and III compared to group I (control) $(\mathrm{p}=0.001)$. The ova of patients with irregular ovulation (both group II and III) failed to reach the size of $11 \mathrm{~mm}$ on day 11 of menstrual cycle (Figure 2 B).

Regarding the dietary history, the intake of carbohydrates and fats was significantly higher in group II and III females, compared to group I females. In addition, the intake of vegetables and fresh fruits in group II and III patients was significantly lower than that of group I females (50\% of Group II and III had only 2 servings of vegetables and fruits, compared to 4 servings in Group I control) . No statistically significant difference in proteins intake was found between the 3 studied groups (Figure 3).

In group I, Kisspeptin level positively correlated with BMI ( $\mathrm{p}=0.02)$. In group II, BMI positively correlated with the body fat $\%$ $(\mathrm{P}<0.001)$, Kisspeptin correlated positively with follicle size. In group III, BMI correlated positively with the body fat $\%$. Body fat $\%$ negatively correlated with kisspeptin level (not the $\mathrm{BMI}$ ), and the follicle size negatively correlated with the BMI (Table 2). 
Table 1: Comparison between the BMI, the age, the waist hip ratio and the waist/height ratio of the 3 studied groups

F: F for ANOVA test, pairwise comparison between each 2 groups was done using Post Hoc Test (Tukey)

$\mathrm{p}$ : $\mathrm{p}$ value for comparing between the three groups

\begin{tabular}{|c|c|c|c|c|c|}
\hline & Group I & Group II & Group III & $\mathbf{F}$ & $\mathbf{p}$ \\
\hline \multicolumn{6}{|l|}{ BMI $\left(\mathrm{Kg} / \mathrm{m}^{2}\right)$} \\
\hline Mean \pm SD & $25.25 \pm 3.83$ & $25.50 \pm 3.70$ & $36.43 \pm 5.4$ & $62.087^{*}$ & $<0.001^{*}$ \\
\hline Significance between groups & \multicolumn{3}{|c|}{$\mathrm{p}_{1}=0.817, \mathrm{p}_{2}<0.001^{*}, \mathrm{p}_{3}<0.001^{*}$} & & \\
\hline \multicolumn{6}{|l|}{ Age (years) } \\
\hline Mean \pm SD & $24.45 \pm 5.46$ & $24.03 \pm 4.63$ & $27.17 \pm 4.80$ & $3.446^{*}$ & $0.036^{*}$ \\
\hline Significance between groups & \multicolumn{3}{|c|}{$\mathrm{p}_{1}=0.954, \mathrm{p}_{2}=0.141, \mathrm{p}_{3}=0.041^{*}$} & & \\
\hline \multicolumn{6}{|l|}{ Waist/hip ratio } \\
\hline Mean \pm SD & $0.79 \pm 0.03$ & $0.9 \pm 0.03$ & $1.03 \pm 0.07$ & $133.386^{*}$ & $<0.001^{*}$ \\
\hline Significance between groups & \multicolumn{3}{|c|}{$\mathrm{p}_{1}<0.001^{*} \mathrm{p}_{2}<0.001^{*}, \mathrm{p}_{3}<0.001^{*}$} & & \\
\hline \multicolumn{6}{|l|}{ Waist/height ratio } \\
\hline Mean \pm SD & $0.49 \pm 0.03$ & $0.59 \pm 0.05$ & $0.71 \pm 0.07$ & $97.627^{*}$ & $<0.001^{*}$ \\
\hline Significance between groups & \multicolumn{3}{|c|}{$\mathrm{p}_{1}<0.001^{*} \mathrm{p}_{2}<0.001^{*}, \mathrm{p}_{3}<0.001^{*}$} & & \\
\hline
\end{tabular}

$\mathrm{p}_{1}$ : $\mathrm{p}$ value for comparing between group I and group II

$\mathrm{P}_{2}$ : $\mathrm{p}$ value for comparing between group I and group III

$\mathrm{p}_{3}$ : $\mathrm{p}$ value for comparing between group II and group III

*Statistically significant at $\mathrm{p} \leq 0.05$

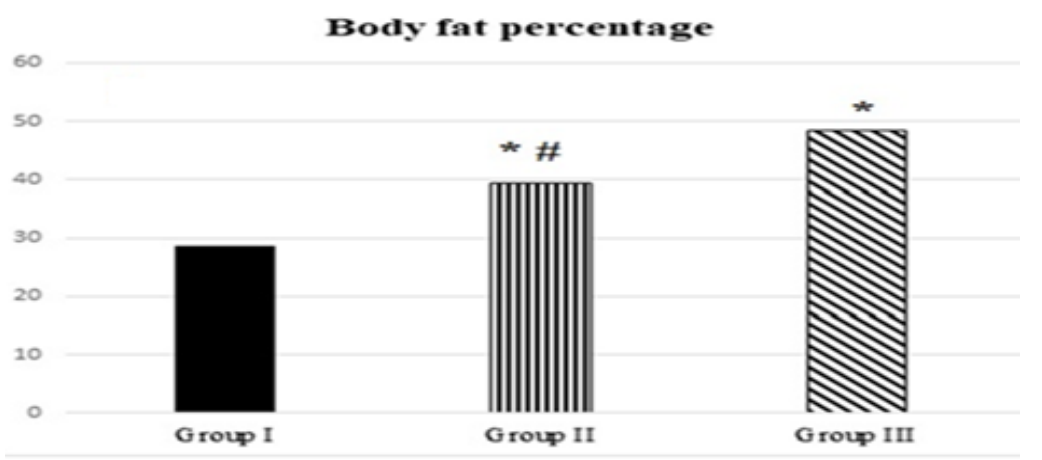

Fig 1: Comparison between the body fat percentage of the 3 studied groups.

* Statistically significant difference with group I.

\# Statistically significant difference with group III

$\mathrm{P}<0.05$ was considered statistically significant.

Serum kisspepetin level

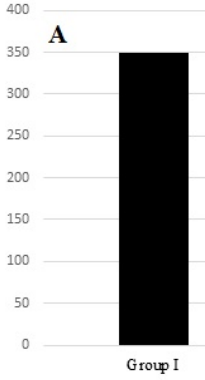

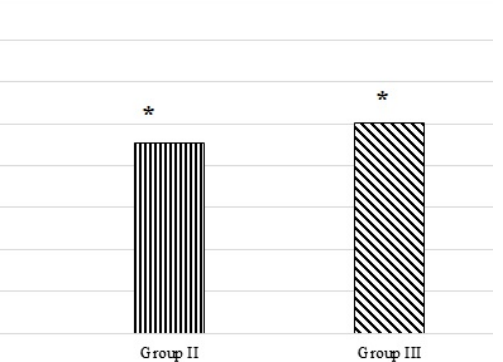

Follicle size at day 11 of menstrual cycle

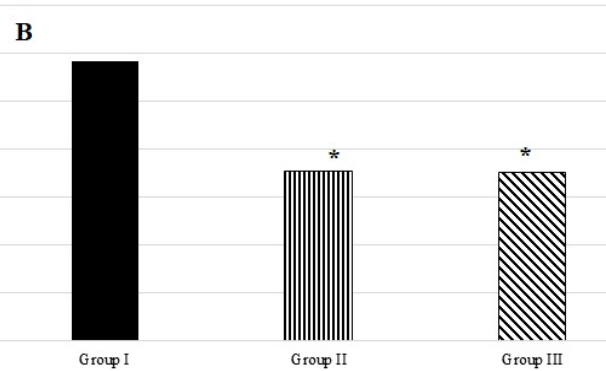

Fig2: Comparison between the serum kisspeptin levels in $\mathrm{pg} / \mathrm{ml}$ and the follicle size in $\mathrm{mm}$ between the 3 studied groups.

* Statistically significant difference with group I. P < 0.05 was considered statistically significant 


\section{Intake of carbohydrates, fats and proteins}

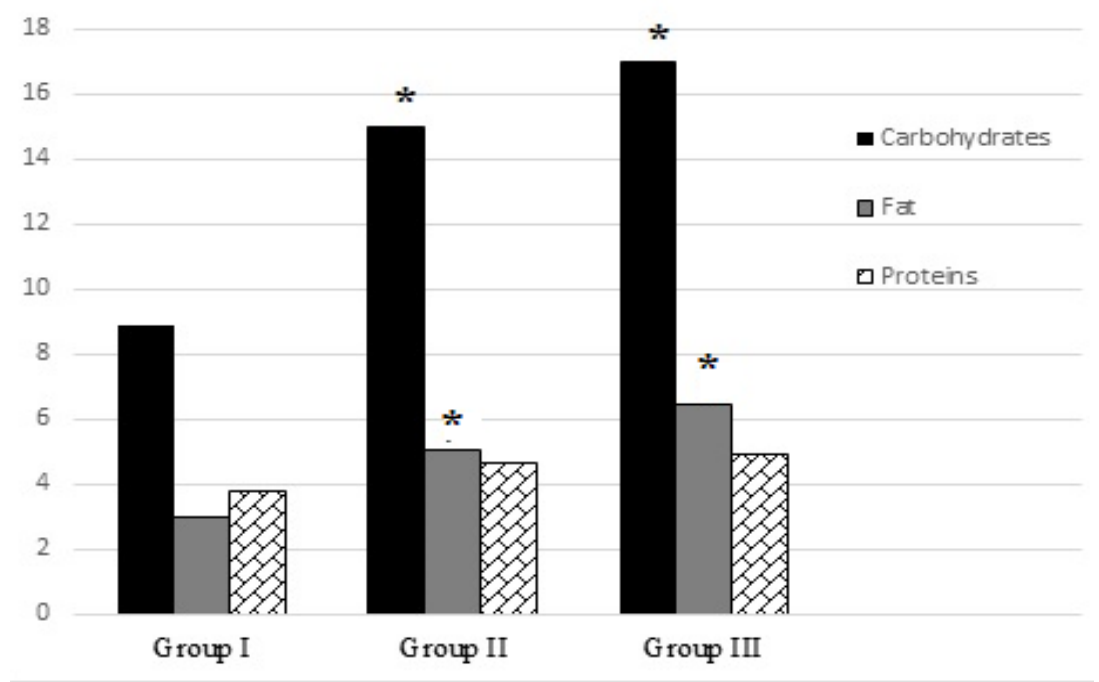

Fig 3: Comparison between the carbohydrates, fat and protein servings in the 3 studied groups.

* Statistically significant difference with group I.

$\mathrm{P}<0.05$ was considered statistically significant

Table 2 : Correlations between some parameters in each group

\begin{tabular}{|l|l|l|}
\hline & \multicolumn{1}{|c|}{$\mathbf{r}_{\mathbf{s}}$} & \multicolumn{1}{|c|}{ P } \\
\hline Group I & \multicolumn{1}{|c|}{} \\
\hline Kisspeptin level versus BMI & $0.516^{*}$ & 0.02 \\
\hline Group II & & \\
\hline BMI versus body fat \% & $0.600^{*}$ & $<0.001$ \\
\hline Kisspeptin versus Follicle size & $0.371^{*}$ & 0.043 \\
\hline Group III & & \\
\hline BMI versus body fat \% & $0.593^{*}$ & 0.001 \\
\hline Body fat \% versus kisspeptin level & $-0.431^{*}$ & 0.018 \\
\hline Follicle size versus BMI & $-0.366^{*}$ & 0.047 \\
\hline
\end{tabular}

$\mathrm{r}_{\mathrm{s}}$ Spearman coefficient $*$ : Statistically significant at $\mathrm{p} \leq 0.05$

\section{Discussion}

In the present study, the mean BMI of group III patients was significantly higher than that of both group I and group II. The BMI was not different between Group I females (having normal cycles) and those belonging to group II (with irregular menstruation but still with BMI <30).

However, the body fat percentage was significantly higher in both group II and III patients compared to group I control. Females with regular menstrual cycles (group I) had a body fat percentage $<32 \%$, while in those with irregular menstruation (both group II and III), it was $>32 \%$. This level of body fat percentage might be the key difference between regular and irregular ovulation. In fact, the American Council on Exercise considers adult females to have excess fat if their body fat percentage equals or is more than $32 \%$, even if their BMI is below 30 [18]. This means that the body fat percentage and not the BMI is the most accurate diagnostic tool of obesity [13]. In the current study, it was the main differentiating factor between females with and without irregular menstruation and disturbed ovulation. This is because the main problem in obesity is having too much fat, not too much weight. In fact, the BMI does not give an indication about the body 
composition, whether the excess weight is fat, muscles or water .

Regarding abdominal adiposity, the waist/hip ratio and the waist to height ratio were both significantly higher in group II and III females than in group I females with regular menstruation. This means that patients with disturbed ovulation had a higher abdominal fat collection, which was detected by either one or more of the applied tests. These findings are in accordance with those of Gonzales (2015) [19] who added that there is an actual link between abdominal obesity and irregular ovulation. It is also worsened by intake of excess glucose and/or saturated fat that may induce an inflammatory state in patients with irregular ovulation .

The ability of excess fat to negatively impact ovulation and menstruation agrees with the findings of Wahab et al. (2018) [20] who demonstrated that the kisspeptin neurons in the hypothalamus are very sensitive to the metabolic state of the body. i.e., in cases of overweight or obesity (positive energy balance) or severe decrease in the body weight (negative energy balance), several changes in the signaling pathway of kisspeptin neurons occur. This is mainly due to direct regulation of the kisspeptin expressing neurons by metabolic hormones .

Kisspeptin level was significantly lower in group II and III, those suffering from disturbed ovulation, compared to group I (control) having normal ovulation. This can be explained by the fact that Kisspeptin was found to show negative correlations with obesity, insulin resistance, and hormones known to affect insulin sensitivity in females [21]. Kisspeptin is a neuropeptide playing a crucial role in modulating of the HPG axis. It acts as a secretagogue of Gn RH [22]. Knowing that the slow rate of $\mathrm{GnRH}$ secretion (less than one pulse /2-3 hours) causes the release of FSH whereas fast rate of $\mathrm{GnRH}$ release (more than 1pulse /hour) causes LH secretion, it is expected that abnormally low levels of kisspeptin would disturb the release of FSH and cause small follicular size and irregular ovulation [21, 22]. Several factors can affect the level of Kisspeptin as stress, circadian variation, immune factors, metabolic factors and gonadal steroids .

However, our results contradict those of Rafique and Latif (2015)[15] who found no statistically significant difference in kisspeptin levels in overweight females compared with normal-weight females. They also found no correlation between any of the anthropometric indices and kisspeptin. The possible explanations of this discrepancy are multiple. First, they used the BMI to discriminate normal weight, overweight and obese females. In the current study, the use of the body fat percentage was able to detect obese females with a body fat percentage higher than 32\% among females even if their body mass index was below 30. Second, the Saudi study was conducted on young Saudi female students in the University of Dammam while in the current work, the mean age of the participating females was higher.

The ova of patients with irregular ovulation (both group II and III) was significantly lower than group I patients and failed to reach the size of $11 \mathrm{~mm}$ on day 11 of ovulation. This finding could be the result of the lower level of kisspeptin measured in both group II and III, compared to the high level of kisspeptin of group I females. The results of the current study are in agreement with 
those of Abbara et al. (2018) [10] who demonstrated the presence of receptors for kisspeptin in the ovary, in theca cells, corpora lutea as well as in the interstitial tissue. This reflects the key role of kisspeptin signaling in the direct control of follicular development, oocyte maturation and ovulation. Any disturbance in the kisspeptin system would have deleterious effects on the functions of the ovaries and may cause infertility. In accordance with our results, Zhai et al. (2017) [23] documented that the peak serum level of kisspeptin may be reached about 48 hours before the sharp surge of estrogen and 72 hours before the surge of LH. This surge coincides with start of estrogen secretion from the dominant follicle. In addition, estrogen induces preovulatory LH surge by a positive feedback mechanism. Therefore, kisspeptin could be considered the earliest trigger of estrogen and LH during the menstrual cycle.

Dietary intake of carbohydrates, fats and proteins give rise to metabolic signals or changes that can modulate the activity of kisspeptin secreting neurons by their effect on anorexigenic and orexigenic neurons [20]. KP neurons control the level of gonadotropin-releasing hormone $(\mathrm{GnRH})$ and adjust the activity of the HPG axis. In the present work, the intake of carbohydrates was significantly higher in group II and III females, compared to group I females. This high intake of carbohydrates, especially simple sugars, stimulates insulin secretion and building of more fat, either subcutaneous or abdominal. On the long run, the patient may develop obesity, insulin resistance and irregular ovulation. Chavarro and his colleagues (2007)[24] found that the risk of ovulatory infertility increases by $78 \%$ in women with higher carbohydrate consumption. On the other hand, low carbohydrate diets not only elicit fast and significant weight loss but also reduced serum insulin and improved insulin sensitivity. The hormonal balance became more healthy: lower free testosterone and higher sex hormone binding globulin. This in turn improved menstrual function and fertility .

The intake of fats was significantly higher in group II females, compared to group I females, specially the intake of hydrogenated vegetable oils. Our results coincide with those of Chavarro et al. (2007) [25] who found that the intake of certain fatty acids augment the risk of developing ovulatory problems. For each $2 \%$ increase in trans fatty acids intake there is a $72 \%$ increased risk of ovulatory infertility, after adjustment for other risk factors. It has been postulated that this may be due to down regulation of peroxisome proliferatoractivated receptor (PPAR) gamma by trans fatty acids in vivo by about $40 \%$. In addition, trans fatty acids increase insulin resistance and inflammatory markers. All these factors have a negative impact on ovulation. However, our results contradict those of other studies that reported the absence of effect of obesogenic diet such as high-fat diet on adult KiSS1 expression $[26,28]$. Researchers postulated that high triglycerides levels and other metabolic dysfunctions associated with obesity and not the degree of body fat and obesity itself modulate kisspeptin expression in the brain.

Regarding the intake of vegetables and fresh fruits, it was significantly lower in group II and III patients than that of group I females. They are great sources of dietary fibers, which play a key role in body health. They maintain prolonged 
satiety. They also modulate the intestinal microflora, which has been found to play a role in development of obesity [29]. In addition, anthocyacines, which are mainly present in fruits especially the peal of apples, blood oranges, strawberries have an antiobesity effect by modulating the genes that control B-oxidation of fatty acids [30]. Nevertheless, epigenetic modulation of kisspeptin was reported [31,32]. Hence, the effects of various dietary elements on ovulation cannot be ignored, not only on the gastrointestinal tract level, but also on a molecular level [33].

\section{Conclusions}

Several studies related obesity and kisspeptin levels to ovulation. High body fat percentage $>32 \%$ in adult females might contribute, in part, in irregular ovulation, even if the BMI is normal. This effect might be mediated via decreasing kisspeptin levels and ovarian follicle size at day 11 of menstrual cycle.

\section{Disclosure Statement}

The authors have no conflicts of interest to declare.

\section{Funding Sources}

This study was self-funded

\section{References}

\section{Mikhael S, Punjala-Patel A, Gavrilova-} Jordan L. Hypothalamic-Pituitary-Ovarian Axis Disorders Impacting Female Fertility. Biomedicines 7(1):5, 2019 .

\section{Clark AM, Thornley B, Tomlinson L,} Galletley C, Norman RJ. Weight loss in obese infertile women results in improvement in reproductive outcome for all forms of fertility treatment. Hum Reprod 13 (6) :1502$5,1998$.

3.Barthelmess EK, Naz RK. Polycystic ovary syndrome: current status and future perspective. Front Biosci (Elite Ed) 6:104-19, 2014.

4.Castro AV, Kolka CM, Kim SP, Bergman RN. Obesity, insulin resistance and comorbidities? Mechanisms of association. Arq Bras Endocrinol Metabol 58(6):600-9, 2014.

5.Orison O. Wool and Richard N. Bergman. Relative fat mass (RFM) as a new estimator of whole-body fat percentage - A crosssectional study in American adult individuals. Sci Rep 8: 10980, 2018

6. Tchernof A, Despres JP. Pathophysiology of human visceral obesity: an update. Physiol $\operatorname{Rev}$ 93(1):359-404, 2013.

7.Wahab F, Atika B, Ullah F, Shahab M, Behr R. Metabolic Impact on the Hypothalamic Kisspeptin-Kiss1r Signaling Pathway. Front. Endocrinol 9:123, 2018.

8.Peng J, Tang M, Zhang BP, Zhang P, Zhong T, Zong T, et al. Kisspeptin stimulates progesterone secretion via the Erk1/2 mitogen-activated protein kinase signaling pathway in rat luteal cells. Fertil Steril 99(5):1436-43.e1, 2013.

9.Jayasena CN, Abbara A, Comninos AN, Nijher GM, Christopoulos G, Narayanaswamy S et al .Kisspeptin-54 triggers egg maturation in women undergoing in vitro fertilization. $J$ Clin Invest.124(8):3667-77, 2014.

10.Abbara A, Vuong L N , Vu N A Ho, Clarke S A , Jeffers L Comninos AN et al .Follicle 
Size on Day of Trigger Most Likely to Yield a Mature Oocyte. Front Endocrinol 9: 193, 2018.

11. Kumar P, Sait SF. Luteinizing hormone and its dilemma in ovulation induction. J Hum Reprod Sci 4(1):2-7, 2011 .

12 . Omani Samani R, Almasi Hashiani A, Razavi M, Vesali, S , Rezaeinejad M, Maroufizadeh S et al. The prevalence of menstrual disorders in Iran: A systematic review and meta-analysis. Int $J$ Reprod Biomed 16(11):665-678, 2018.

13.De Lorenzo A, Soldati L, Sarlo F, Calvani M, Di Lorenzo N, Di Renzo L . New obesity classification criteria as a tool for bariatric surgery indication. World $J$ Gastroenterol 22(2): 681-703, 2016.

14. Gruson E, Montaye M, Kee F, Wagner A, Bingham A, Ruidavets JB et. al . Anthropometric assessment of abdominal obesity and coronary heart disease risk in men: the PRIME study. Heart 96(2):136-40, 2010 .

15 .Rafique N, Latif R. Serum kisspeptin levels in normal and overweight Saudi females and its relation with anthropometric indices. Ann Saudi Med 35(2):157-60, 2015.

16. Franz MJ, Barr P, Holler H, Powers MA, Wheeler ML, Wylie-Rosett J. Exchange lists: revised 1986. J Am Diet Assoc 87(1):2834, 1987. Erratum in: $J$ Am Diet Assoc 87(8):1103, 1987. PMID: 3794130.

17. Kirkpatrick LA, Feeney BC.A simple guide to IBM SPSS statistics for version 20.0.Student ed.Belmont, Calif, Wadsworth, Cengage Learning ;2013 18.https://woman.thenest.com/american-councilexercise-body-fat-percentage-16636.html (last accessed 10/6/2021)

19.González F. Nutrient-Induced Inflammation in Polycystic Ovary Syndrome: Role in the Development of Metabolic Aberration and Ovarian Dysfunction. Semin Reprod Med 33(4):276-86, 2015.

20. Wahab F, Atika B, Ullah F, Shahab M, Behr

R. Metabolic Impact on the Hypothalamic Kisspeptin-Kiss1r Signaling Pathway. Front Endocrinol 9:123, 2018.

21.Kolodziejski PA, Pruszynska-Oszmalek E, Korek E, Sassek M, Szczepankiewicz D, Kaczmarek P, et al. Serum levels of spexin and kisspeptin negatively correlate with obesity and insulin resistance in women. Physiol Res 67(1):45-56, 2018.

22. Tsutsumi R, Webster NJ. GnRH pulsatility, the pituitary response and reproductive dysfunction. Endocr J 56(6):729-37, 2009.

23. Zhai J, Ding L, Zhao S, Li W, Sun Y, Su S, et al. Kisspeptin: a new marker for human pre-ovulation. Gyn Endocrinol 33(7):560-3, 2017.

24. Chavarro JE, Rich-Edwards JW, Rosner B, Willett WC. A prospective study of dairy foods intake and anovulatory infertility. Hum Reprod 22(5):1340-7, 2007.

25. Chavarro JE, Rich-Edwards JW, Rosner BA, Willett WC. Dietary fatty acid intakes and the risk of ovulatory infertility. Am J Clin Nutr 85(1):231-7, 2007.

26. Lie ME, Overgaard A, Mikkelsen JD. Effect of a postnatal high-fat diet exposure on puberty onset, estrous cycle regularity, and 
kisspeptin expression in female rats. Reprod Biol 13(4):298-308, 2013.

27. Luque RM, Kineman RD, Tena-Sempere M. Regulation of hypothalamic expression of KiSS-1 and GPR54 genes by metabolic factors: analyses using mouse models and a cell line. Endocrinol 148(10):4601-11, 2007.

28. Quennell JH, Howell CS, Roa J, Augustine RA, Grattan DR, Anderson GM. Leptin deficiency and diet-induced obesity reduce hypothalamic kisspeptin expression in mice. Endocrinol 152(4):1541-50, 2011.

29.Petraroli M, Castellone E, Patianna V, and Esposito S. Gut Microbiota and Obesity in Adults and Children: The State of the Art. Front Pediatr 9: 657020, 2021.

30. Castro-Barquero S, Lamuela-Raventos RM, Domenech M, Estruch R. Relationship between Mediterranean Dietary Polyphenol Intake and Obesity. Nutrients 10(10), 1523, 2018.
31.Wyatt AK, Zavodna M, Viljoen JL, Stanton JA,Gemmell NJ, Jasoni C. Changes in methylation patterns of kiss1 and kiss1r gene promoters across puberty. Genet Epigenet 5:51-62, 2013.

32. Motti ML , Meccariello R. Minireview: The Epigenetic Modulation of KISS1 in Reproduction and Cancer . Int J Environ Res Public Health 16(14): 2607, 2019.

33.Navarro VN. Metabolic regulation of kisspeptin - the link between energy balance and reproduction. Nat Rev Endocrinol 16, 407-20, 2020. 